\section{Patrón gammagráfico "superscan" en la osteomalacia hipofosfatémica asociada a Tenofovir en un paciente con infección por VIH}

\section{Resumen}

El tratamiento antirretroviral de gran actividad (TARGA) constituye uno de los avances más importantes en el manejo de los pacientes con infección por el virus de la inmunodeficiencia humana (VIH). Sin embargo, su uso a largo plazo conlleva el riesgo de aparición de enfermedades óseas como osteporosis, osteonecrosis y osteomalacia. Uno de los fármacos más utilizados dentro de los esquemas de TARGA es el tenofovir disoproxil fumarato (TDF), un análogo de nucleótido inhibidor de la transcriptasa inversa que tiene buen perfil de seguridad y tolerancia, pero que ha sido asociado con el desarrollo de osteomalacia hipofosfatémica $(\mathrm{OMH})$. Presentamos el caso de un varón de 56 años con infección crónica por VIH que desarrolló dolor óseo generalizado y debilidad durante el tratamiento con TDF, detectándose en el estudio analítico hipofosfatemia y aumento de su nivel basal de fosfatasa alcalina, con fosfaturia y calciuria normales. La radiografía mostraba aplastamientos vertebrales dorsales y la gammagrafía ósea reveló una hipercaptación difusa compatible con patrón "superscan" metabólico. Este patrón ha sido descrito de forma infrecuente en la OMH asociada a TDF, por lo que realizamos una revisión de los casos previamente publicados.

Palabras clave: gammagrafía ósea; patrón superscan; osteomalacia hipofosfatémica; tenofovir; terapia antirretroviral de gran actividad; virus de la inmunodeficiencia humana.
Liudmila Valentina Maldonado Romero', Walter Alberto Sifuentes Giraldo', Javier Bachiller Corral' ${ }^{1}$, Fernando Dronda Núñez ${ }^{2}$

\footnotetext{
1 Servicio de Reumatología. Hospital Universitario Ramón y Cajal.

2 Servicio de Enfermedades Infecciosas. Hospital Universitario Ramón y Cajal.
}

Correspondencia:

Dra. Liudmila Valentina Maldonado Romero

” liudvalen@yahoo.com 


\section{Scintigraphy "superscan" pattern in Tenofovir-associated hypophosphatemic osteomalacia in a patient with HIV infection}

\section{Abstract}

Highly active antiretroviral therapy (HAART) is one of the most important advances in the management of patients infected with human immunodeficiency virus (HIV). However, long term use of these drugs carries the risk of bone diseases such as osteporosis, osteonecrosis and osteomalacia. Tenofovir disoproxil fumarate (TDF), a nucleotide analog reverse-transcriptase inhibitor, is one of the agents most frequently used in the HAART schemes due to its good safety and tolerance profile, but it has been associated with the development of hypophosphatemic osteomalacia (HOM). We report the case of a 56 year old male with chronic HIV infection who developed generalized bone pain and weakness during treatment with TDF, and laboratory tests detected hypophosphatemia and increased levels alkaline phosphatase, with fosfaturia and calciuria within normal ranges. Radiography showed dorsal compression fractures and bone scintigraphy revealed diffuse increased uptake of tracer compatible with a metabolic "superscan" pattern. This pattern has been infrequently reported in TDF associated-HOM, so we make a review of previously published cases.

Key Words: bone scintigraphy; superscan pattern; hypophosphatemic osteomalacia; tenofovir, highly active antiretroviral therapy; human immunodeficiency virus.

\section{Introducción}

Los pacientes con infección por el virus de la inmunodeficiencia humana $(\mathrm{VIH})$ desarrollan frecuentemente enfermedades óseas, incluyendo osteoporosis, osteonecrosis y osteomalacia. Aunque la etiología de estos trastornos es multifactorial, relacionándose tanto con la infección por VIH como con las comorbilidades asociadas, un factor importante a considerar es la toxicidad atribuible al tratamiento antirretroviral de gran actividad (TARGA) [1]. Entre estos fármacos se encuentra el tenofovir disoproxil fumarato (TDF), un análogo de nucleótido inhibidor de la transcriptasa inversa ampliamente utilizado debido a su perfil de seguridad y tolerancia. Sin embargo, ha sido asociado con nefrotoxicidad, disminución de la densidad mineral ósea y osteomalacia hipofosfatémica $(\mathrm{OMH})[2,3]$. Presentamos el caso de un paciente con infección por VIH que desarrolló un cuadro de $\mathrm{OMH}$ durante el tratamiento con TDF que mostraba un patrón de "superscan" en la gammagrafía ósea, el cual ha sido descrito con poca frecuencia en este tipo de pacientes $[4,5,6]$. 


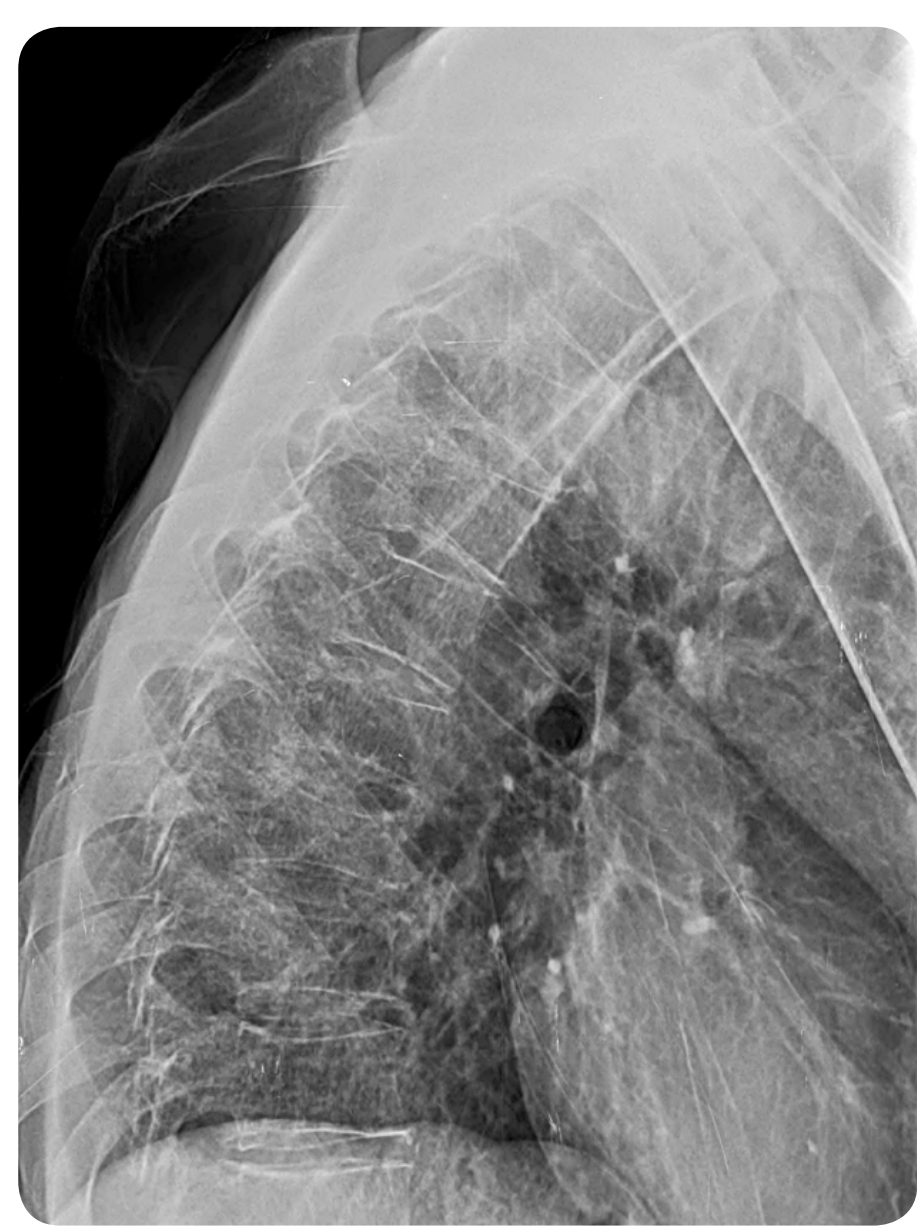

Figura 1. Radiografía lateral de columna dorsal con aplastamientos anteriores en D8 y D9.

\section{Caso Clínico}

Un varón de 56 años consultó en octubre de 2012 por dolor óseo generalizado y debilidad de 8 meses de evolución. Tenía antecedentes de infección por VIH estadio C3 diagnosticada 23 años antes, cirrosis secundaria a infección por el virus de la hepatitis C, tabaquismo, abuso de drogas intravenosas, duodenopancreatectomía cefálica por carcinoma neuroendocrino duodenal e intestino corto secundario a resección yeyunal. Desde junio de 2003 se le administraba TARGA que incluía TDF, fosamprenavir, emtricitabina y ritonavir, manteniendo carga viral < 35 copias $/ \mathrm{mL}$ y niveles de CD4+ 275/ $\mathrm{mm}^{3}$. Recibía además suplementos de calcio y vitamina D3. A la exploración presentaba caquexia y dolor a la presión sobre eminencias óseas, pero la fuer-

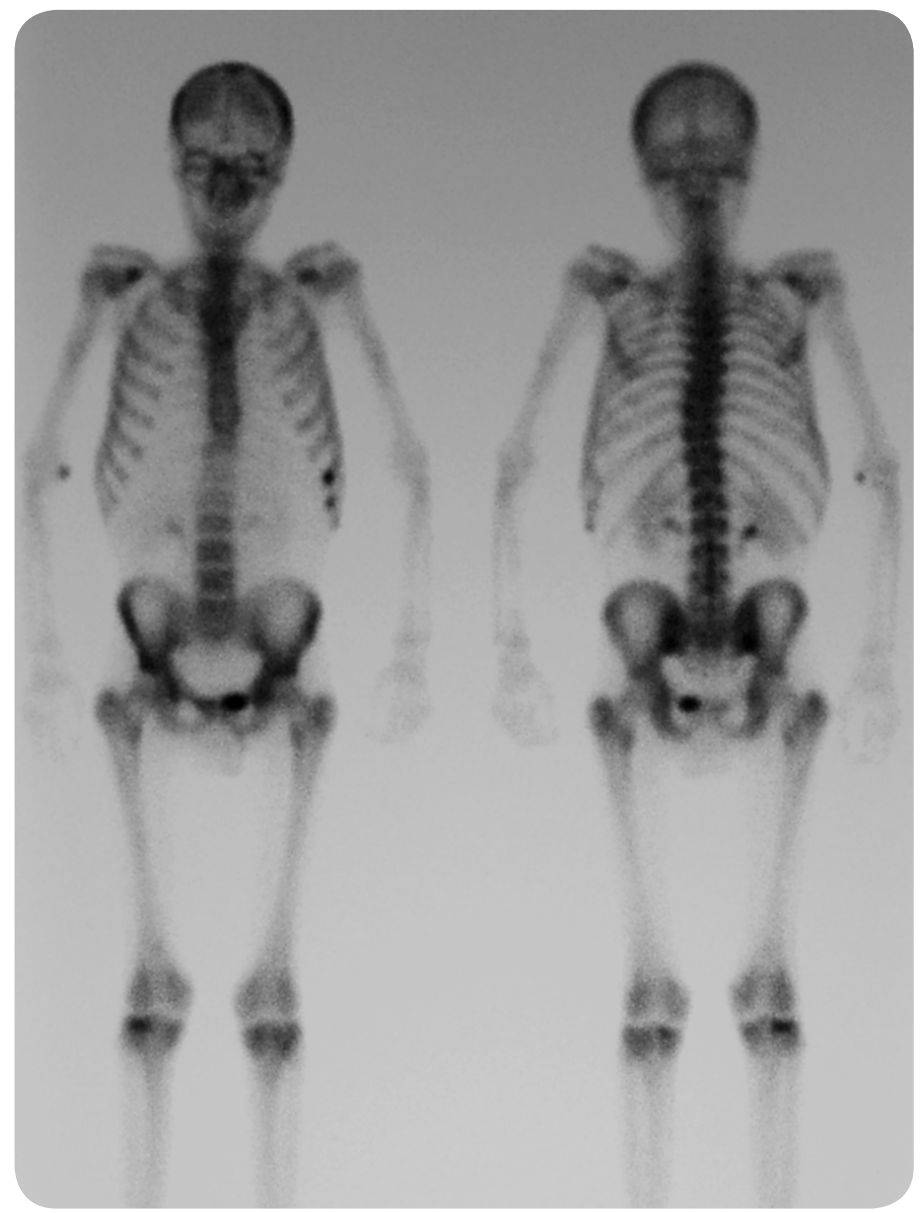

Figura 2. Gammagrafía ósea de cuerpo completo en la que se observa aumento generalizado, difuso, y homogéneo de la captación, más intensa a nivel periarticular y axial, con escasa visualización de las siluetas renales, compatible con patrón "superscan".

za muscular se encontraba conservada. La analítica mostraba creatinina $0,85 \mathrm{mg} / \mathrm{dL}$, calcio $8,8 \mathrm{mg} / \mathrm{dL}$, fósforo $1,5 \mathrm{mg} / \mathrm{dL}$, fosfatasa alcalina (FA) $217 \mathrm{U} / \mathrm{L}$ (nivel basal $180 \mathrm{U} / \mathrm{L}$ ), 25(OH) vitamina D3 12,7 ng/ $\mathrm{mL}, 1,25(\mathrm{OH})$ vitamina $\mathrm{D} 324,5 \mathrm{pg} / \mathrm{mL}$, parathormona 41,8 pg/mL, calciuria 217 mg/24h, fosfaturia $948 \mathrm{mg} / 24 \mathrm{~h}$, y reabsorción tubular de fosfato (RTP) $67,58 \%$. Se evidenciaron aplastamientos vertebrales anteriores en D8 y D9 (Fig. 1) con osteoporosis a nivel axial (T-score -3) y en cuello femoral (T-score -4). La gammagrafía ósea con Tc99m reveló un aumento difuso y homogéneo de la captación tanto en esqueleto axial como apendicular, compatible con patrón "superscan" metabólico (Fig. 2), diagnosticándose de $\mathrm{OMH}$. Se investigó una probable 
recidiva del tumor neuroendocrino, con resultados negativos de endoscopia, marcadores tumorales y pruebas de imagen. Se atribuyó el desarrollo de $\mathrm{OMH}$ al tratamiento con TDF, siendo suspendido y reemplazado con inhibidores de la proteasa (darunavir más ritonavir). Se incrementó el aporte de calcio y vitamina $D$, y se pautó suplemento de fosfato, con mejoría progresiva de la clínica y normalización de la fosfatemia, calcemia, RTP y $25(\mathrm{OH})$ vitamina D3 en el control analítico realizado a las 6 semanas. La FA disminuyó, pero se mantuvo por encima del límite superior normal debido a la hepatopatía de base. No se pudo realizar seguimiento gammagráfico posterior ya que el paciente falleció pocos meses después debido a encefalopatía hepática.

\section{Discusión}

El desarrollo de $\mathrm{OMH}$ durante el tratamiento con TDF es raro, habiéndose publicado 32 casos hasta la fecha [3]. Su prevalencia se estima en 0,5\% de los pacientes tratados [7]. El mecanismo por el que este fármaco puede ocasionar $\mathrm{OMH}$ parece ser una alteración en el túbulo contorneado proximal que conduce a pérdida renal de fosfato $[4,8]$. Algunos pacientes pueden presentar tubulopatía severa compatible con síndrome de Fanconi (glucosuria, aminoaciduria, hipercalciuria, hipouricemia, hipopotasemia, acidosis metabólica) [3-7]. Se ha descrito, además, que el TDF puede interferir con la 1- $\alpha$ hidroxilación de la vitamina D3 y actuar directamente sobre los osteoclastos alterando su función [1].

Clínicamente, la OMH cursa con dolor óseo (con o sin fracturas patológicas), debilidad muscular y la analítica muestra aumento de FA, hipofosfatemia, fosfaturia inapropiadamente normal o aumentada, con calciuria normal y niveles de vitamina D3 normales o bajos [4, 8]. El tiempo desde el inicio de TDF hasta el diagnóstico de $\mathrm{OMH}$ es variable, habiéndose descrito desde las 4 semanas hasta más allá de los 5 años de tratamiento $[3,6]$. La frecuencia de fracturas en estos pacientes depende de la severidad de la $\mathrm{OMH}$, habiéndose descrito que hasta un $79 \%$ de los casos las desarrollan, mientras que un 20\% pueden presentar pseudofracturas [16]. En pocos casos se ha realizado el estudio histopatológico óseo, encontrándose acumulación de osteoide con captación baja o indetectable de tetraciclinas (reflejando ausencia de mineralización), así como disminución de osteoclastos y niveles bajos de reabsorción ósea [3].

El uso concomitante de TDF y ritonavir, un inhibidor de la proteasa, pueden aumentar las posibilidades de desarrollar $\mathrm{OMH}$ ya que este último aumenta las concentraciones séricas de TDF [14]. También se han publicado casos con adefovir, un análogo de nucleótido inhibidor de la transcriptasa inversa utilizado para el tratamiento de la Hepatitis B [10, 11]. Otras causas que se deberían considerar en el diagnóstico diferencial de la OMH asociada a TDF son los tumores productores de proteína relacionada con la parathormona (PTHrP) (denominada "Osteomalacia oncogénica"), la OMH idiopática del adulto, la OMH hereditaria y los síndromes de malabsorción [9]. En nuestro caso no hubo evidencia de recidiva del tumor neuroendocrino, lo que aleja el origen tumoral. La malabsorción por el síndrome de intestino corto y la hepatopatía de base podrían haber contribuido al desarrollo de $\mathrm{OMH}$, pero consideramos que no son los factores principales debido a que la osteomalacia por malabsorción generalmente es debida al déficit de $25(\mathrm{OH})$ vitamina D3, por lo que sus niveles son más bajos ( $<10 \mathrm{ng} /$ $\mathrm{mL}$ ) y se asocia a hipocalciuria (< $100 \mathrm{mg} / 24 \mathrm{~h}$ ) [17], dato ausente en nuestro caso. Además, el paciente desarrolló este cuadro a pesar de recibir suplementos de calcio y vitamina D3, y presentó mejoría tras la retirada de TDF, lo cual apoya su rol causal.

El diagnóstico de $\mathrm{OMH}$ se basa en los síntomas clínicos, las alteraciones metabólicas y los hallazgos 
Tabla 1. Características demográficas y clínicas de los casos de osteomalacia hipofosfatémica asociada al tratamiento con tenofovir en la infección por VIH con patrón "superscan"

\begin{tabular}{|c|c|c|c|c|c|c|c|c|}
\hline $\begin{array}{l}\text { Caso } \\
\text { (Ref.) }\end{array}$ & $\begin{array}{l}\text { Sexo/ } \\
\text { Edad }\end{array}$ & $\begin{array}{c}\text { Estadio } \\
\text { VIH }\end{array}$ & $\begin{array}{l}\text { CD4+(cél } / \mu \mathrm{L}) / \\
\text { carga viral } \\
\text { (copias } / \mathrm{mL} \text { ) }\end{array}$ & TARGA & $\begin{array}{l}\text { Duración } \\
\text { de TDF } \\
\text { (meses) }\end{array}$ & Presentación clínica & Tratamiento & Evolución \\
\hline $1[4]$ & $\begin{array}{l}M \\
46 \text { a }\end{array}$ & C3 & $128 /<50$ & $\begin{array}{l}\text { EFV, } \\
\text { FTC, } \\
\text { TDF }\end{array}$ & 22 & $\begin{array}{c}\text { Dolor osteomuscular } \\
\text { Debilidad } \\
\text { Síndrome de Fanconi }\end{array}$ & $\begin{array}{l}\text { Suspensión de } \\
\text { TDF }\end{array}$ & $\begin{array}{c}\text { Resolución clínica } \\
\text { y analítica a los } \\
3 \text { meses }\end{array}$ \\
\hline $2[5]$ & $\begin{array}{l}\mathrm{H} \\
37 \mathrm{a}\end{array}$ & C3 & $360 / I D$ & $\begin{array}{l}\text { LPV, } \\
\text { RTV, } \\
\text { TDF }\end{array}$ & 19 & $\begin{array}{l}\text { Dolor óseo } \\
\text { Síndrome de Fanconi }\end{array}$ & $\begin{array}{l}\text { Suspensión de } \\
\text { TDF y LPV } \\
\text { Suplementos de } \\
\text { Ca, VitD3, P } \\
\text { y HCO3 }\end{array}$ & $\begin{array}{l}\text { Datos no } \\
\text { disponibles }\end{array}$ \\
\hline $3[6]$ & $\begin{array}{l}M \\
48 \text { a }\end{array}$ & ND & $267 / 30000$ & $\begin{array}{l}\text { EFV, } \\
\text { AZT, } \\
3 T C, \\
\text { IDV, } \\
\text { d4T, } \\
\text { TDF }\end{array}$ & 1,5 & $\begin{array}{l}\text { Dolor óseo } \\
\text { Síndrome de Fanconi }\end{array}$ & $\begin{array}{l}\text { Suspensión } \\
\text { de TDF } \\
\text { Suplementos } \\
\text { de VitD3 }\end{array}$ & $\begin{array}{c}\text { Resolución } \\
\text { clínica y analítica. } \\
\text { Ausencia de } \\
\text { hipercaptación } \\
\text { al año }\end{array}$ \\
\hline $\begin{array}{l}\text { Caso } \\
\text { actual }\end{array}$ & $\begin{array}{l}\mathrm{H}, \\
56 \mathrm{a}\end{array}$ & $\mathrm{C} 3$ & $275 /<35$ & $\begin{array}{l}\text { FPV, } \\
\text { FTC, } \\
\text { RTV, } \\
\text { TDF }\end{array}$ & 104 & $\begin{array}{c}\text { Dolor óseo } \\
\text { Debilidad } \\
\text { Fracturas vertebrales }\end{array}$ & $\begin{array}{l}\text { Suspensión } \\
\text { de TDF y LPV }\end{array}$ & $\begin{array}{c}\text { Resolución clínica } \\
\text { y analítica a las } \\
6 \text { semanas }\end{array}$ \\
\hline
\end{tabular}

Abreviaturas: Ref., referencia; VIH, virus de la inmunodeficiencia humana; $H$, hombre; $M$, mujer; ID, indetectable; TARGA, tratamiento antirretroviral de gran actividad; EFV, efavirenz; FTC, emtricitabina; TDF, tenofovir; LPV, lopinavir, RTV; ritonavir, AZT, zidovudina; 3TC, lamivudina; IDV, indinavir; d4T, estavudina; FPV, fosamprenavir; Ca, calcio; VitD3, calcitriol; P, fósforo; HCO3, bicarbonato.

de las pruebas de imagen como la gammagrafía ósea o la resonancia magnética [3]. Los patrones gammagráficos más frecuentemente en los casos publicados en la literatura son aquellos con captaciones compatibles con fracturas por insuficiencia o pseudofracturas $[2,3,9]$. Sin embargo, en 3 de ellos se encontró un aumento generalizado de la incorporación del trazador en todo el esqueleto con ausencia o muy débil visualización de las siluetas renales, patrón conocido como "superscan" [4, $5,6,19]$. Las características clínicas de dichos casos se resumen en la Tabla 1. El patrón "superscan" es característico de enfermedades óseas metabólicas como la osteomalacia, hiperparatiroidismo, osteopetrosis y enfermedad de Paget, pero también se debe considerar en el diagnóstico diferencial a las metástasis óseas (mama, estómago, próstata, pulmón), trastornos hematológicos (leucemia, linfoma, mielofibrosis, macroglobulinemia de Waldenstrom), enfermedades inflamatorias (artritis reumatoide, espondilitis anquilosante), intoxicación por aluminio, displasia fibrosa e hipertiroidismo [18]. En uno de los casos publicados de $\mathrm{OMH}$ asociada a TDF se realizó control gammagráfico al año de suspender el fármaco, evidenciándose la desaparición de este patrón [6].

Si se sospecha de OMH y/o nefrotoxicidad, la interrupción de TDF y la suplementación oral de fosfatos es el enfoque adecuado para prevenir un daño renal permanente. Las recomendaciones generales para la $\mathrm{OMH}$ incluyen suplementos de calcio (1000- 


\section{ACTA REUMATOLÓGICA}

1500 mg/día), calcifediol (1000-2000 Ul/día) y fosfato 1-3 g/día repartidos en 3 a 4 dosis, además la coadministración de calcitriol (0.5-2 $\mu \mathrm{g} / \mathrm{día})$ parece útil para aumentar la absorción de fosfato en el intestino y acelerar la recuperación $[2,14,15]$.

En conclusión, aunque la $\mathrm{OMH}$ es infrecuente en la infección por HIV es importante su conocimiento y sospecha clínica. Según las directrices actuales, en los pacientes tratados con TDF se recomienda la monitorización periódica (cada 3-6 meses) de la tasa de filtración glomerular, niveles de fósforo sérico y FA, especialmente si existen comorbilidades asociadas $[7,12,13]$. Así mismo, cuando estos pacientes presenten dolor osteomuscular, debilidad, baja densidad mineral ósea o fracturas se debería descartar la presencia de OMH. La gammagrafía ósea puede ser de gran utilidad en estos casos, demostrando tanto la presencia de fracturas por insuficiencia como el patrón "superscan" metabólico. 


\section{Referencias}

1. Cotter AG, Powderly WG. Endocrine complications of human immunodeficiency virus infection: hypogonadism, bone disease and tenofovir-related toxicity. Best Pract Res Clin Endocrinol Metab. 2011; 25:501-15.

2. De Socio GV, Fabbriciani G, Massarotti M, Messina S, Cecchini E, Marasini B. Hypophosphatemic Osteomalacia Associated with TDF: a Multidisciplinary Approach is Required. Mediterr J Hematol Infect Dis. 2012; 4:e2012025.

3. Mateo L, Holgado S, Mariñoso ML, Pérez-Andrés R, Bonjoch A, Romeu J, Olivé A. Hypophosphatemic osteomalacia induced by tenofovir in HIV-infected patients. Clin Rheumatol. 2014, http:// dx.doi.org/ 10.1007/s10067-014-2627-x (en prensa).

4. Pérez-Rivera AÁ, Sáez $P$, León E, Lozano de León-Naranjo F. HIV infected women with intense bone and muscular pain and general weakness. Enferm Infecc Microbiol Clin. 2011; 29:308-10.

5. Torres MV, García T, Gómez MV, González F, Gambí N, Castilla $S$, et al. Utilidad de la gammagrafía ósea en el diagnóstico de osteomalacia secundaria en un paciente en tratamiento con tenofovir. Rev Esp Med Nucl. 2006; 25:103-6.

6. Di Biagio A, Rosso R, Monteforte P, Russo R, Rovetta G, Viscoli. Whole body bone scintigraphy in tenofovir-related osteomalacia: a case report. J Med Case Report. 2009; 3:8136.

7. Woodward CL, Hall AM, Williams IG, Madge S, Copas A, Nair $D$, et al. Tenofovir-associated renal and bone toxicity. HIV Med. 2009; 10:482-7.

8. Tjen-A-Looi A, Naseer SN, Worthing AB, Timpone JG, Kumar PN. Hypophosphatemic Osteomalacia Associated with Tenofovir Use in HIV Infected Patients: A Case Series and Review of the Literature. J AIDS Res. 2012; S4:008.
9. Parsonage MJ, Wilkins EG, Snowden N, Issa BG, Savage MW. The development of hypophosphataemic osteomalacia with myopathy in two patients with HIV infection receiving tenofovir therapy. HIV Med. 2005 Sep;6(5):341-6.

10. Palermo A, Strollo R, Papalia R, et al. Endocr Pract. 2014 Aug 22:1-15

11. Jeong HJ, Lee JM, J Bone Metab. 2014. Feb; 21 (1):76-83.

12. European AIDS Clinical Society. Web site. http://www.european aidsclinicalsociety.org/guidelines/g2.htm. Accessed November, 2011.

13. GuptaSK, Eustace JA, Winston JA, Boydstun II, AhujaTS, Rodriguez RA et al (2005) Guidelines for the management of chronic kidney disease in HIV-infected patients: recommendations of the HIV Medicine Association of the Infectious Diseases Society of America. Clin Infect Dis 40(11):1559-1585.

14. L. Mateo, S. Holgado et al. Hypophosphatemic osteomalacia induced by tenofovir in HIV-infected patients. Clin Rheumatol. DOI 10.1007/s10067-014-2627-x.

16. Reuss-Borst, M A. Metabolic bone disease osteomalacia.Z rheumatol. 2014 May;73 (4):316-22

17. Laia Gifre, et al. Osteomalacia revisited: A report on 28 cases. Clin Rheumatol. 2011; 30:639-645

18. Basha B, Rao DS, Han ZH, Parfitt AM. Osteomalacia due to vitamin $D$ depletion: a neglected consequence of intestinal malabsorption. Am J Med. 2000; 108:296-300.

19. Buckley O, O'Keeffe S, Geoghegan T, Lyburn ID, Munk PL, Worsley D, et al. $99 \mathrm{mTc}$ bone scintigraphy superscans: a review. Nucl Med Commun. 2007; 28:521-7.

20. Monserrat Minoves Font (2010). Técnicas de imagen: Estudios Isotópicos. Manual de Enfermedades Óseas. Sociedad Española de Reumatología (pp 101). Lugar de edición: Editorial médica Panamericana. 
Opina sobre este artículo:

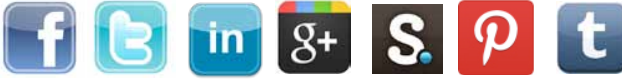

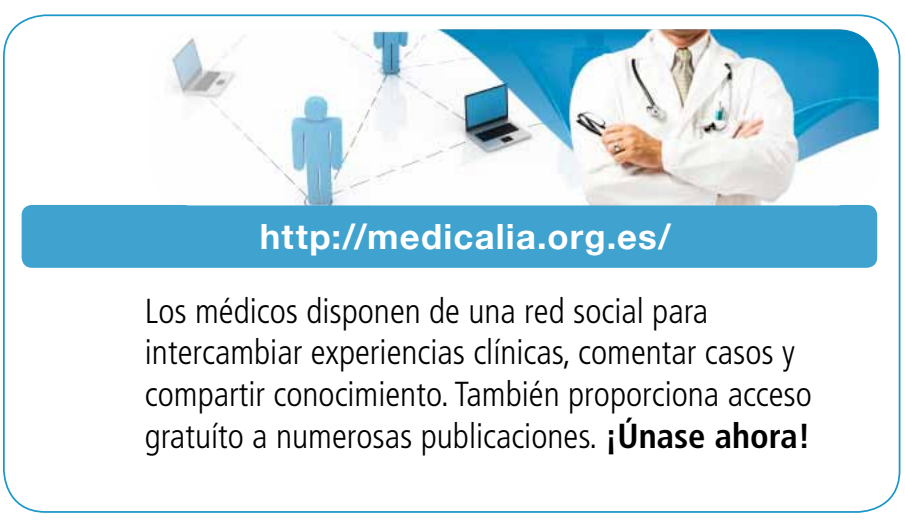

\section{Publish with iMedPub}

\section{http://www.imed.pub}

Acta Reumatológica es una revista que tiene por fin la difusión de estudios clínicos relacionados con aspectos prácticos del diagnóstico, tratamiento y seguimiento de pacientes con patología reumatológica, de estudios epidemiológicos relacionados con patología inflamatoria y musculoesquelética de presentación común o infrecuente en la práctica clínica tanto en población adulta como pediátrica, de casos clínicos de patología poco habitual o de presentaciones inhabituales de patología frecuente, de imágenes didácticas e ilustrativas en reumatología y del estado actual e innovación en la formación especializada en reumatología. 